\title{
Representing a Combat ID Analysis Tool within an Agent Based Constructive Simulation
}

\author{
David Dean \\ Dstl \\ dfdean@dstl.gov.uk \\ Beejal Mistry \\ Dstl \\ bmistry@dstl.gov.uk
}

\author{
Alasdair Vincent \\ Dstl \\ avincent@dstl.gov.uk \\ Aleem Hossain \\ Dstl \\ ahossain@dstl.gov.uk \\ Mink Spaans \\ TNO \\ Mink.Spaans@tno.nl
}

\begin{abstract}
Motivation - This paper describes an approach for the development of a model which represents complex human factors relationships relating to Combat Identification (Combat ID). Research approach - The human factors relationships were incorporated within an experimental Agent Based Model (ABM), using an integrated approach to analysis and experimentation. Research limitations/Implications - This is ongoing work, and is currently limited in application to a single decision maker within a static environment. Originality/Value - The approach has the potential to provide a new way of representing cognitive errors, and humans SA (Situation Awareness) development. Take away message - Within the context of Combat ID, the approach has enabled cognitive errors, and the impact of human bias on SA development to be modelled within an experimental agent based constructive simulation
\end{abstract}

\section{Keywords}

Human Factors, Combat ID, Agent Based Model, Constructive Simulation, Situational Awarenes.

\section{INTRODUCTION}

The authors have been studying the representation of Combat Identification (Combat ID) ${ }^{1}$ within combat models and analysis tools. One of the key observations that came from this work is the overriding impact of human factors, particularly those based around cognitive science, on the outcome of the Combat ID process.

This led to the development of the Integrative Combat Identification Entity Relationship (INCIDER) model, an analysis tool that represents a Combat ID 'encounter'" The logic and processes that are included in INCIDER include aspects which are generally applicable to decision-making. Therefore the extension of the model to represent human error mechanisms within other decision-making processes would be a logical next step.

This paper includes a case study describing the implementation of a specific Combat ID analysis tool within a constructive simulation, illustrating a process used to bring all of these strands together as part of an iterative, multilayered approach to model development.

There have been many attempts to introduce human factors into constructive simulations. In the past these have been based upon simple stochastic parameters, intended to represent imperfections in the combat and decision-making processes. These tended to represent quality parameters and trigger points at which behaviour would change. Examples include:

- TRAINING and EXPERIENCE factors which increase- or decrease- detection and engagement ranges, and ENGAGEMENT EFFECTIVENESS (essentially improving the single shot kill probability).

\footnotetext{
${ }^{1}$ UK military doctrine defines Combat Identification as "The process of combining situational awareness, target identification, specific tactics, training and procedures to increase operational effectiveness of weapon systems and reduce the incidence of casualties caused by friendly fire." (Ministry of Defence 2006).

${ }^{2}$ INCIDER defines an encounter as being the process of a single decision-maker detecting and identifying an unknown object or entity on the battlespace as described in Dean and Handley (2006). 
- MORALE and FEAR factors which, when combined with a UNIT QUALITY attribute, lead to either PANIC and RETREAT Or EXUBERANCE and a degree of LOSS OF CONTROL.

Although such approaches are valid in their attempts to represent aspects of human behaviour and are perfectly acceptable ways of generating certain representations of human performance, they are limited in their fidelity and cannot be used to represent more complex cognitive processes.

The INCIDER model, which is the basis of the case study described in this paper, was developed as a means of predicting the outcomes of Combat ID encounters. It is a complex and unique tool which predicts the outcome of an identification process undertaken by a single decision-maker observing a single unknown entity. This work has required extensive consideration of the nature of human factors and possible methods for modelling these factors. The INCIDER model has been validated by a number of Synthetic Environment based experiments, which have provided an empirical link between its representations of cognitive behaviour and behaviour recorded from virtual world observations.

A limitation of INCIDER is its inability to deal with many-on-many encounters. This severely reduces the fidelity of its representations of Situational Awareness (SA). The logical next step is to incorporate the core behaviour of INCIDER within an Agent Based Model. This paper will describe work undertaken collaboratively by $\mathrm{Dstl}^{3}$ and $\mathrm{TNO}^{4}$ to implement a representation of the INCIDER model within the NetLogo 5 agent based modelling tool (Wilensky 1999). This work has required the development of a number of novel human factors representations, and has followed an integrated approach to analysis and experimentation.

\section{A PROCESS TO DEVELOP HUMAN FACTORS RELATIONSHIPS}

The complexity and uniqueness of the INCIDER decision model led to the development of an integrated analysis and experimentation process. This facilitated progression from a defined problem to the generation of a human factors representation, embedded within a constructive simulation. This process is summarised in Figure 1 . The 5 coloured boxes represent the core activities undertaken during INCIDER development; these were supported by the tasks shown in grey boxes linking into them.

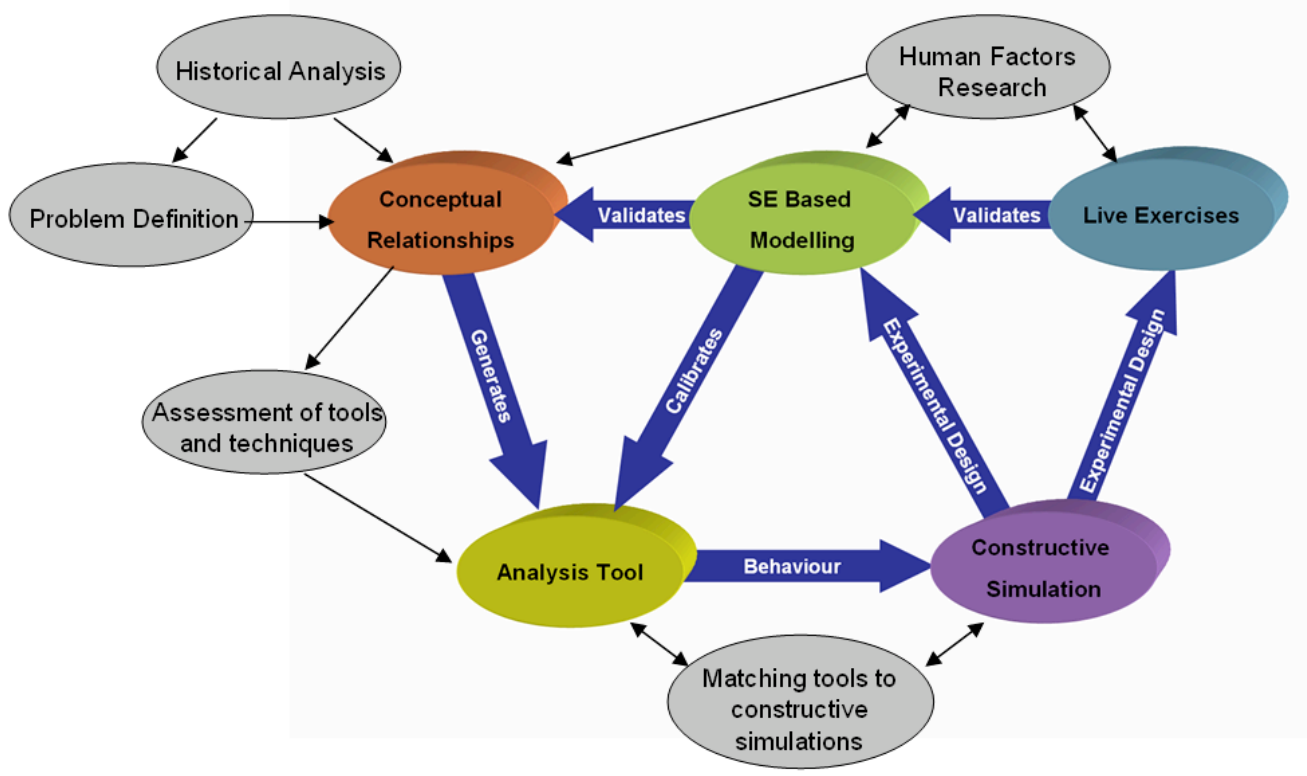

Figure 1 - Process components for Human Factors model development

\footnotetext{
${ }^{3}$ Defence Science and Technology Laboratory (Dstl) - Part of the UK Ministry of Defence (MOD) responsible for providing advice on Science and Technology.

${ }^{4}$ Toegepast-Natuurwetenschappelijk Onderzoek TNO. An English translation is: the Netherlands Organisation for Applied Scientific Research.

${ }^{5}$ NetLogo is a freeware modelling tool, developed by Northwestern University.
} 
The process is iterative, and each activity, particularly those in the central section, should be revisited a number of times. The intention is that all of the different areas are developed in parallel. This ensures that holistic decisions are taken during the development processes, and the consequences of implementing decisions on exploitation routes will be better understood.

The process nominally starts ${ }^{6}$ with problem definition. This can be supported by historical analysis, and will certainly involve the input of stakeholders; generally military customers and end-users. Once the problem has been defined, a set of conceptual relationships to support the human factors representations will emerge; these will essentially consist of a set of human factors along with their definitions, metrics and interrelationships. The problem will be fed from human factors research (and body of knowledge) in particular targeted experiments, often using Synthetic Environments (SE).

The conceptual relationships can be used as a source from which to derive a number of analysis tools, each representing different facets of human behaviour. In the case of INCIDER, a model was developed that represented the process performed by a single decision-maker during a single encounter. In order to assess the 'fitness-for-purpose' of the analysis tool, a number of iterative experiments were undertaken which in turn led to a number of different tools and techniques being assessed for applicability.

Both the analysis tool and conceptual relationships will need to be validated, and it is almost unavoidable that the validation process will result in a revision of both the model and the analysis tool. An effective method adopted by the Combat ID research was to undertake initial validation of INCIDER using SEs, including adaptations of commercial computer games (so-called 'serious games'). It is important to note that any SE to be utilised must be validated against the real world before any inferences about real world behaviour can be made; typically, this can be done through live exercises or historical evidence.

The next step is to investigate exploitation routes, particularly by looking at potential applications within constructive simulations. This requires matching between the host constructive simulation and analysis tool, which will enable both tool and simulation to be assessed for fit. If an existing tool is to be utilised, it must be acknowledged that there will be strict limitations on the ability of the model to represent the desired parameters.

Such limitations must be identified early since it is possible that they will be severe enough to render the representation useless. In any case, the representation within the constructive simulation must be assessed against the validation experiment or other empirical data as soon as is practical in order to identify implementation problems early on.

An alternative approach is to opt for a new, bespoke development. This, however, involves a high level of risk. The Dstl INCIDER team, in association with colleagues from TNO, opted for a phased approach to implementation:

- $\quad$ Phase one - an experimental agent based model was developed in NetLogo to test theories and identify new areas of development and requirements. The model developed, although crude, could be applied to analysis applications. This development is described later in this paper.

- Phase two - integrate INCIDER concepts into a modified constructive simulation, the Close Action Environment (CAEn). Phase one is being used to de-risk future development of CAEn. It is intended to use lessons learned within NetLogo to improve the future development of CAEn.

The partial (or full) development of a constructive simulation representation will lead to new requirements for validation, verification and research, feeding back into the initial stages and continuing the iterative cycle.

\section{A FRAMEWORK FOR INSTANTIATING HUMAN FACTORS IN COMBAT MODELS}

In order to support the process of developing Human Factors relationships, it is useful to consider how each stage can be used to develop elements of a supporting framework. Gathering these components together allows some of the important relationships between these areas to be explored. The framework is illustrated in Figure 2 and described in more detail in Dean et al. (2008).

\footnotetext{
${ }^{6}$ Due to the iterative nature of the process, it is possible to start elsewhere and work around the cycle to the problem definition task - this could be the case for pure human factors research which has been commissioned with no defined customer question.
} 

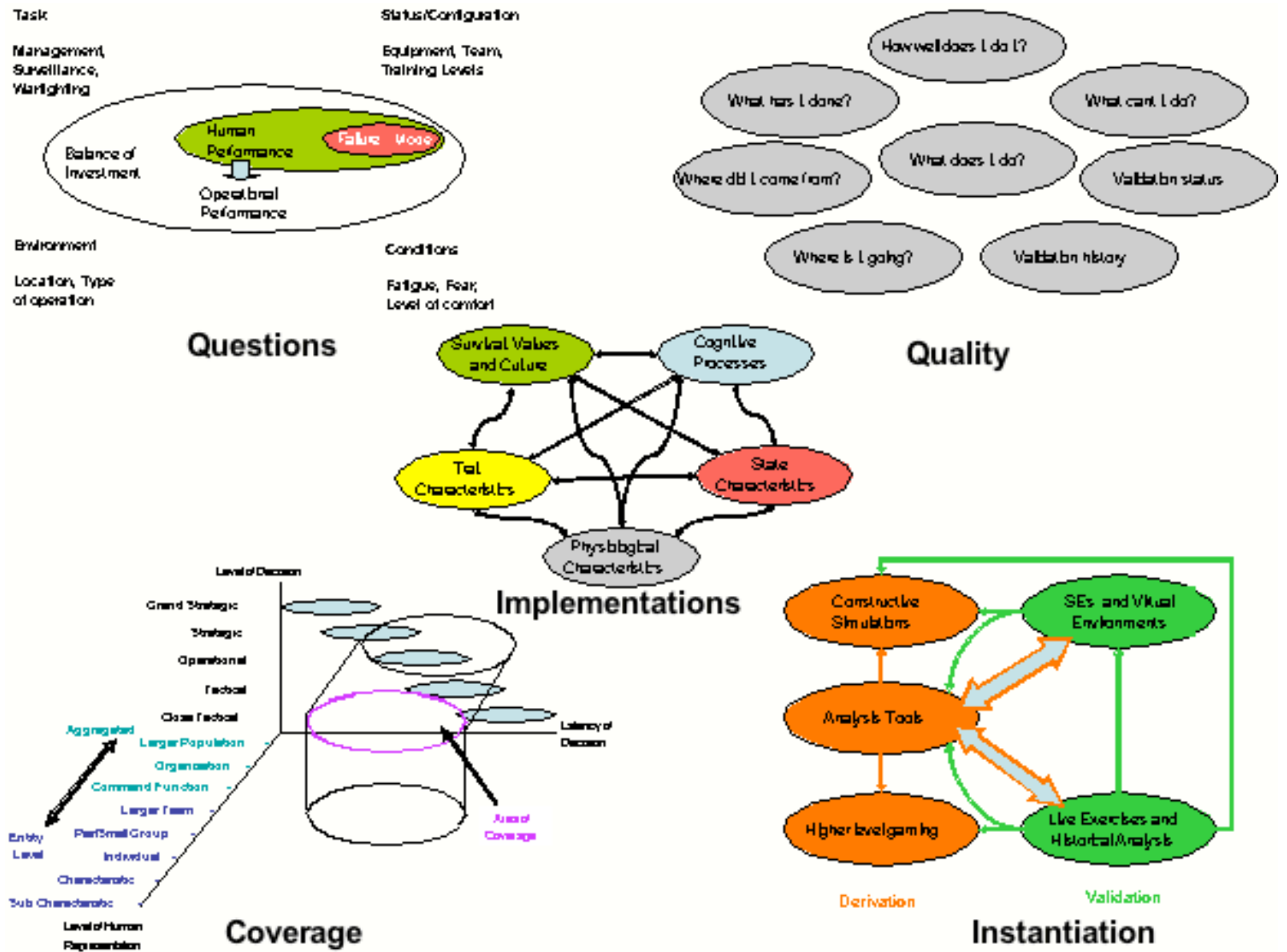

Quality

Figure 2 - A framework for instantiating human factors in combat models

The framework is composed of six areas ${ }^{7}$ which describe the problem space; aspects of human behaviour and performance to be represented; context; and solution space. The areas are summarised in the list below:

1. Types of question - What questions do the models and representations need to address? These can include investment decisions, assessment of operational effectiveness, computation of reaction times etc. It is important to understand what questions are to be addressed in order to assess the suitability of an analysis approach, and the fidelity required.

2. Types of human representation - Is the model representing individuals, teams, or other types of abstracted behaviour? The answer may be all three, but each type will give rise to different assumptions and demonstrate human characteristics in different ways.

3. Levels of human decision representation - There are four main areas of representation that constructive simulations need to address; Strategic, Operational, Tactical and Close Tactical. Associated with these are different decision times, and different types of interaction with encompassing and interfacing systems.

4. Types of implementation - What type of analysis tool or constructive simulation is the human representation to reside within? In particular, what constraints does it impose upon the human representation?

5. Types of characteristic to be represented -Which human aspects are to be represented? This could represent anything from cognitive processes to physiological performance.

6. Quality - What is the status of the representation? Has it been validated? What degree of confidence can be attributed to its use?

\footnotetext{
${ }^{7}$ Note that 'Level of Decision', and 'Level of Human Representation' have been combined under the 'Coverage' category in the figure above.
} 


\section{A CASE STUDY - IMPLEMENTING INCIDER WITHIN AN AGENT BASED SIMULATION}

This section will describe ongoing research to develop a working version of a complex human factors representation within the NetLogo agent based modelling environment. The representations that are achievable within it are relatively crude; however it is an extremely useful test-bed for identifying and exploring functionality that can be used to de-risk CAEn development, whilst at the same time leading to the production of a simple simulation tool.

\section{INCIDER Model Overview}

The INCIDER model was developed to answer Balance of Investment (BOI) questions for the UK Ministry of Defence; specifically, to look at the BOI between Situational Awareness (SA), Target Identification (TID) systems, and Tactics, Techniques and Procedures (TTPs; e.g. doctrine and training). The INCIDER model was developed based on an initial investigation including historical analysis, military judgement and psychological literature review ${ }^{9}$ The model has two main components:

- The INCIDER relationships model is a repository containing more than 70 parameters relating to the Combat ID decision-making process, grouped under physical, human and operational categories. The conceptual model represents area 5 of the framework, the characteristics to be represented.

- The INCIDER encounter model is an analysis tool which represents the process undertaken by a single decision-maker identifying a single unknown entity. In particular it represents the following:

- $\quad$ The distance between the entities at initial detection;

- $\quad$ The real identity of the unknown entity;

- $\quad$ The sensor systems and information sources available to the decision-maker;

- Human characteristics of the decision-maker (Personality, Experience, Stress, Fatigue);

- The level of confidence that the decision-maker needs in order make a decision;

- The decision-maker's belief and preconceptions (what they are expecting to see, based on prior briefing and history up to the event of interest).

The INCIDER models are summarised in Figure 3. The encounter model process compares new information about the unknown entity (from sensors and information sources) with a representation of preconception (based on a mixture of pre-mission briefing and gut feel). It then iteratively obtains more information by using the different sources available and by moving closer to the unknown entity. This process continues until either a decision is reached, or until a timeout condition is reached, indicating that the decision-maker was unable to declare an identification decision.

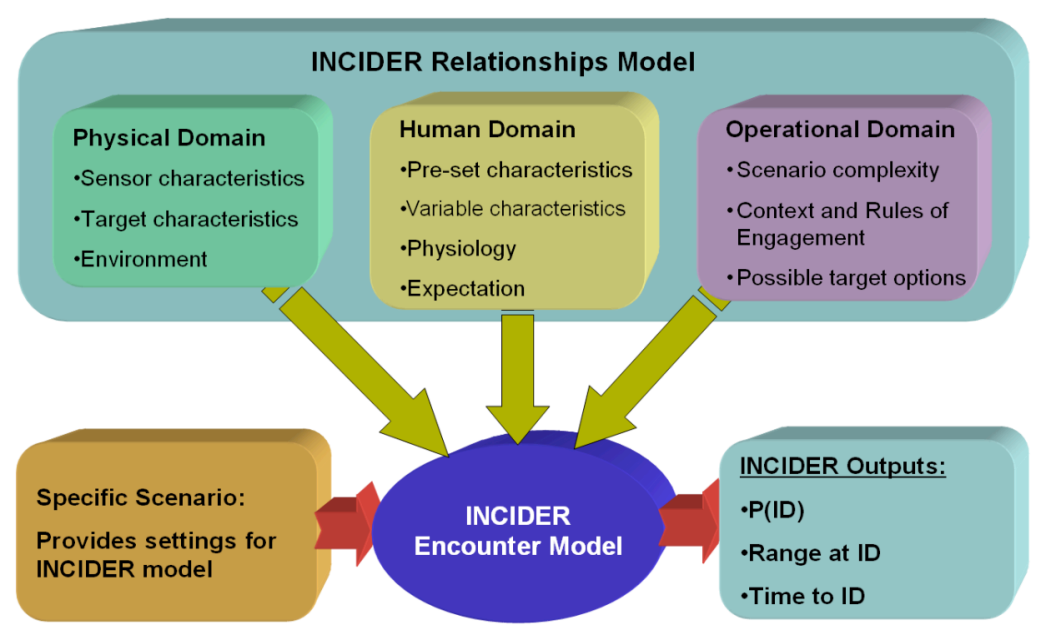

Figure 3 - The INCIDER model

Running INCIDER multiple times enables statistics to be gathered on probability of correct identification, probability of incorrect identification, probability of no decision, time taken to identify, and the range at which identification takes place (i.e. the separation between the decision-maker and the unknown entity).

\footnotetext{
${ }^{9}$ For example Newell (1990); Nofi (2000); Eysenck and Keane (1999); Reason (2000); and Klein (1989)
} 


\section{Implementation within the NetLogo Tool}

A tool was required which could rapidly be used to develop simple representations of behaviour, and investigate the effect of a large number of different variations in SA, TID, HF, and TTP within different operational contexts on mission level combat effectiveness and fratricide. An analysis of suitable tools highlighted NetLogo as being a suitable development environment (Wilensky 1999).

In order to examine the effects of a large range of different variations in SA, the use of Data Farming ${ }^{15}$ (Brandstein and Horne 1998) was adopted. Data Farming allows for the investigation of huge numbers of scenarios by the use of efficient experimental designs. The Data Farming practices were engineered in cooperation with the SEED Centre ${ }^{16}$.

The NetLogo model represents a single agent that moves through the environment encountering, and identifying surrounding objects. The allegiance of objects can either be part of the enemy forces (red), neutral (green), or friendly (blue), and can be of type 'person', 'car', or 'tank'.

\section{Initialisation}

The model initialises by automatically generating a ground truth of red, green, and blue objects (this uses a random distribution and is therefore data farmable). Currently, this is done by defining three random centre points on the X-axis (one for red, green and blue). A triangular probability distribution is then initialized around this point with random $\mathrm{Y}$ values. For each run, the centres of each side will differ, enabling random amounts of overlap between sides, and hence the automatic generation of a large number of random scenarios.

The model now generates an overlay of preconception. This represents what the agent thinks is in the ground truth ${ }^{17}$ (this can also be considered as its belief, or SA). The agent's belief of a certain spot in the environment is defined as a normalised triplet (for each point on the grid, the belief in the identity at that location of each of the allegiance types is

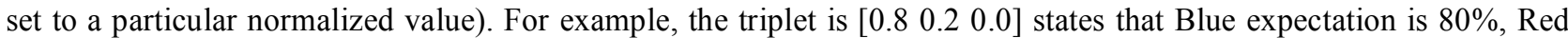
expectation is $20 \%$, Green expectation is $0 \%$. The mechanism for defining centre points is also used for the distribution of beliefs. This distribution of beliefs is parameterized, and thus data farmable.

\section{Agent Behaviour}

After initialization, the simulation begins, with the agent exploring the ground truth, identifying objects, and updating its SA. Exploring the ground truth is currently implemented as a pseudo-random movement through the environment ${ }^{19}$. The agent can detect, recognise, and identify (DRI) objects depending upon range. The DRI values are set as range dependent probabilities, and vary depending upon the ground truth (the values of each are data farmable).

On initial detection, the agent will enter into a representation of the INCIDER decision-making process, and can either decide on identity, or move closer. Identification is based on a comparison of the preconception grid, and identification probability. Identification decisions take place once the decision threshold (another data farmable variable) is exceeded by the current level of belief.

\section{Representation of SA}

An addition to INCIDER behaviour implemented by the NetLogo model was to introduce the notion of Global SA and Local SA. This creates a distinction between (Global) SA about the entire environment and (Local) SA about the direct surroundings of the agent ${ }^{20}$.

The size of the local SA and the granularity of the global SA are parameterized (and thus data farmable). The local SA is updated each time new sensor information is accepted or as a result of moving. When the agent moves, the local SA

\footnotetext{
${ }^{15}$ Data Farming is a method that applies high performance computing to modelling in order to examine and understand the landscape of potential simulated outcomes, enhance intuition, find surprises and outliers, and identify potential options.

${ }^{16}$ The SEED Center for Data Farming http://harvest.nps.edu/

${ }^{17}$ This essentially represents a decision-maker's prior belief, typically as a result of information gained from a premission briefing.

${ }^{19}$ Fully random movement could bring the agent into an undesirable loop, not exploring the whole ground truth.

${ }^{20}$ Global SA may be viewed as a part of history, or Long Term Memory (LTM), and Local SA as Working Memory (WM).
} 
grid moves with it, keeping the agent centred. As a result of the move, some cells will be removed from the local SA and new cells are added, taking the belief distribution of the global SA cell as its initial belief (see Figure 6).

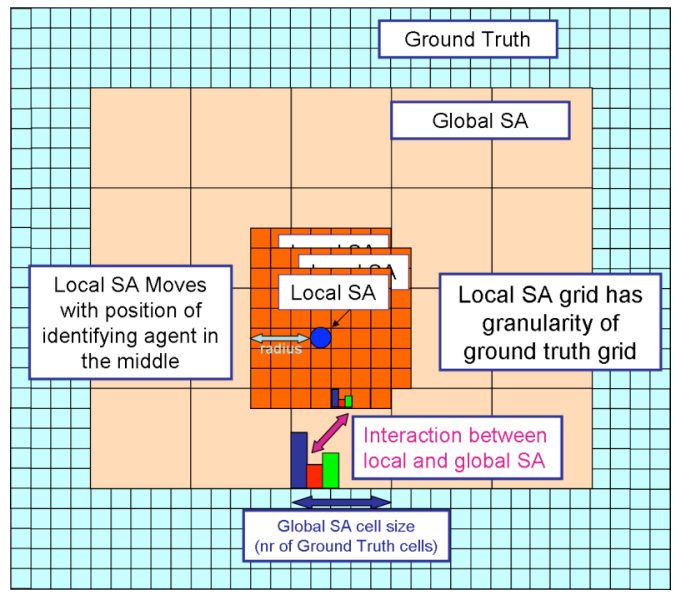

Figure 6: Global SA and Local SA

After encountering an object, an agent who has a detailed Global SA updates its belief over a small area, whereas an agent with a less detailed Global SA updates its belief over a larger area. The same holds, to some extent, in real world (battle space) environments. In case of open warfare one could roughly localize the enemy. However, within urban warfare behind each wall, door, or window there might be an ally, a citizen, or an enemy. A number of implementations of SA were investigated, in particular how global SA can be updated from local SA and observed ground truth.

\section{Representation of Preconception}

The INCIDER study identified human factors confirmatory bias concepts ${ }^{21}$ as among the most important influences on Combat ID. In order to model these concepts, Information Acceptance Curves are used to represent the agent's willingness to accept/reject sensor input.

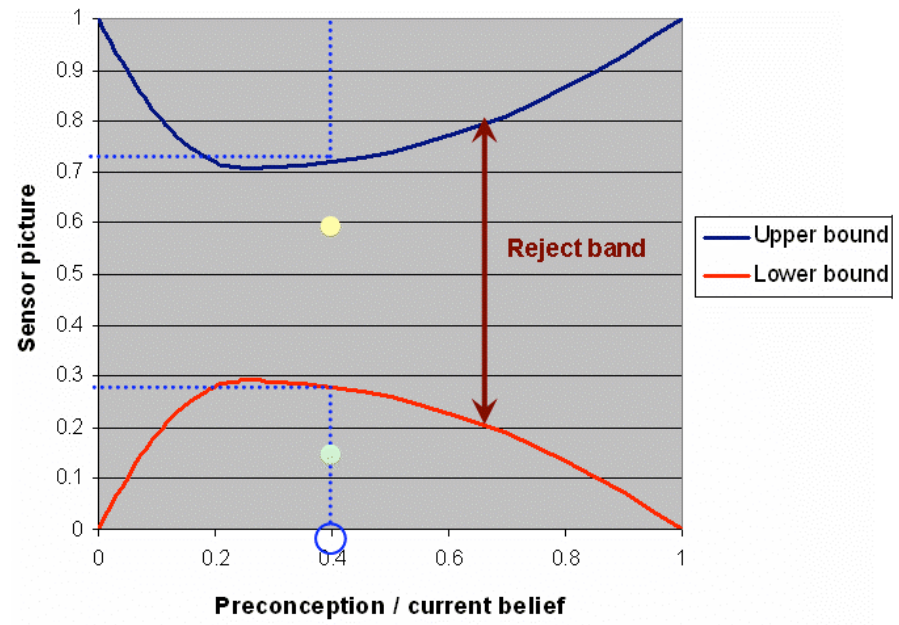

Figure 7: Information acceptance curves

Figure 7 shows an example of the curves used to represent confirmatory bias. They act as a band reject filter, with sensor inputs that fall between the curves being ignored in favour of the preconception. The curves work by rejecting new information with weak belief when preconception is strongly believed, but accepting new information when preconception is weak. In Figure 8, a preconception score of 0.4 translates to a lower bound of $\sim 0.29$, and an upper bound of $\sim 0.72$. Any information between these values will be rejected, and information outside of these values will be accepted, and form the decision maker's new belief.

${ }^{21}$ Confirmatory bias is the tendency of individuals to reject new information because it does not agree with their prior beliefs (see Eysenck and Keane (1999)). 
As the agent becomes more receptive to new information (or more open minded) the reject band between the two sets of curves decreases. As the agent becomes less willing to accept new information (or close-minded) the opposite is true, and the agent's preconception determines the outcome of the identification process leading to a higher potential for misidentification.

\section{SUMMARY}

This paper has described a framework and an associated process for the representation of human factors within constructive simulations, which has proved to be extremely useful during the development, validation and instantiation of the INCIDER model. The migration of the INCIDER model to constructive simulations has developed a number of unique representations of human decision-making and SA within the context of Combat ID.

The use of the NetLogo experimental prototype has proved to be an extremely powerful and flexible technique for the rapid development of human factors representations and has been used to de-risk future development of the CAEn model. It is anticipated that the human representations within the CAEn model could evolve to a level where it can provide a flexible representation of combat ID. This will allow for its application to a variety of analysis tasks.

The NetLogo tool remains as an adaptable developmental testbed, and has the potential to continue to drive the requirements of future simulation development. However it too could have applications as an analysis tool to address a range of focussed analysis questions.

\section{CONCLUSIONS}

The representation of Human Factors within models and analysis is an extremely complex and demanding endeavour. The framework provides a set of checkpoints to characterise and contextualise such representations. This has the potential to reduce the risks, time and costs of model development as part of a Systems Engineering approach to model development.

The agent based model representation described by the case study has made huge progress in an extremely short space of time. The prototypes are still being refined and expanded, and it is planned to have much more complex behaviour represented within the models. The data farming approach has proved to be an excellent way to de-risk model development. A number of novel representations of SA have also been developed which can potentially be applied to a variety of different applications.

\section{REFERENCES}

Brandstein, A.G. and G. E. Horne (1998). Data farming: A Meta-Technique for Research in the 21st Century. In F. G. Hoffman and G. E. Horne (Eds.), Maneuver Warfare Science 1998, pp. 93-99. Marine Corps Combat Development Command: Quantico,VA.

Dean, D. and A. Handley (2006). Representing the Human Decision Maker in Combat Identification. In Proceedings of the 11th International Command and Control Research and Technology Symposium (ICCRTS'06), 26-28 September, Cambridge.

Dean, D., A. Vincent, B. Mistry, M. Spaans and P. Petiet (2008). An Architecture for Placing the Human at the Centre of the Constructive Simulation. In Proceedings of the 13th International Command and Control Research and Technology Symposium (ICCRTS’08), 17-19 June, Bellevue, WA.

Eysenck, M. W. and M. T. Keane (1999). Cognitive Psychology: A Student's Handbook (Third ed.). Hove: Psychology Press.

Klein, G. (1989). Recognition-Primed Decisions. In W. B. Rouse (Ed.) Advances in Man-Machine Systems Research, Vol. 5, pp. 47-92. Greenwich, CA: JAI Press.

Ministry of Defence (2006). United Kingdom Glossary of Joint and Multinational Terms and Definitions. Joint Doctrine Publication 0-01.1

Newell, A. (1990). Unified Theories of Cognition. Cambridge, MA: Harvard University Press.

Nofi, A. A. (2000). Defining and Measuring Shared Situation Awareness. Technical Report CRM D0002895.A1/Final, Center for Naval Analyses.

Reason, J. (2000). Human Error: Models and Management. British Medical Journal 320(7237):768-770.

Wilensky, U. (1999). NetLogo. http://ccl.northwestern.edu/netlogo/. Center for Connected Learning and ComputerBased Modeling, Northwestern University, Evanston, IL. 